\title{
Severe Recurrent Achalasia Cardia Responding to Treatment of Severe Autoimmune Acquired Haemophilia
}

\author{
H. Al-Jafar M. Laffan S. Al-Sabah M. Elmorsi \\ M. Habeeb F. Alnajar
}

Amiri Hospital, Kuwait City, Kuwait

\section{Key Words}

Recurrent achalasia cardia $\cdot$ Autoimmune acquired haemophilia Cross-reacting autoantibodies

\begin{abstract}
Acquired haemophilia $A$ and severe acquired achalasia are both very rare conditions with unknown aetiology. Haemophilia $A$ is a haemorrhagic disease induced by deficiency or malfunction of coagulation factor VIII. Congenital haemophilia is an inherited disease transmitted by the mother through $\mathrm{X}$-linked inheritance and primarily affects males. However, acquired haemophilia $\mathrm{A}$ is a serious, sudden-onset, autoimmune disease that affects either sex. In addition, achalasia is a disease of the oesophagus caused by abnormal function of the nerves and muscles. It causes swallowing difficulties due to the inability of the lower oesophageal sphincter to relax during swallowing, leading to dysphagia, regurgitation and chest pain. In this report, we describe the case of a patient with severe, newly diagnosed, acquired haemophilia A with long-standing, recurrent achalasia; the achalasia had recurred 3 times despite complete and proper surgical fixation. Acquired haemophilia $A$ is treated with immunosuppressive therapy. High-dose steroid therapy was administered for 7 months, during which the patient responded well; moreover, the achalasia did not recur for more than 2 years. The response of the achalasia to immunosuppressive therapy suggests that achalasia may be an autoimmune disorder and that there may be an association between both diseases. The findings of the present case suggest that achalasia may favourably respond to steroid therapy as a first-line treatment prior to surgery.
\end{abstract}




\section{Introduction}

Haemophilia may be an acquired or congenital disorder. The congenital forms of haemophilia A and B are caused by low levels of coagulation factor VIII (FVIII) and coagulation factor IX, respectively. These protein factors are essential for the generation of thrombin, which is required to stabilise the primary platelet plug and thus help control bleeding. FVIII deficiency may also be caused by autoantibodies directed against the FVIII molecule, leading to acquired haemophilia. Congenital haemophilia manifests with spontaneous haemorrhage in muscle and joints and, rarely, in the skin and soft tissues. Acquired haemophilia manifests with extensive subcutaneous extravasations and mucosal haemorrhages in the gastrointestinal tract, urinary tract and female genital tract, and extensive, painful, intramuscular hematomas [1]. Severe haemophilia A affects approximately 1 in 10,000 males, and severe haemophilia B affects approximately 1 in 50,000 males [2]. Acquired haemophilia is less common: evaluation of the frequency in the UK identified an unbiased cohort of 172 patients with an incidence of 1.48 per million per year. Achalasia is a rare disease of the nerves and muscles of the oesophagus caused by failure of the oesophageal muscles to relax during swallowing and the inability of the lower oesophageal sphincter to open. This difficulty in swallowing prevents food from passing into the stomach [3]. Achalasia comprises 3 types, based on the severity of the symptoms: hypermotile, hypomotile and amotile. The disease can occur at any age, with a similar rate in men and women, but it is usually diagnosed between 25 and 60 years of age. It is characterised predominantly by dysphagia to solids and liquids, bland regurgitation and chest pain [4]. It has an annual incidence of approximately 1 in 100,000 and a prevalence rate of 1 in 10,000. Complications of achalasia include weight loss, aspiration pneumonia and oesophagitis caused by irritation from collection of food and fluids in the oesophagus. There is also a risk of cancer of the oesophagus in patients with long-standing achalasia. Some reports have described achalasia as an autoimmune disorder [5], and there is evidence suggesting that different autoimmune disorders may be associated in the same patient. In this case report, we describe a patient with acquired haemophilia A associated with acquired achalasia. We explore the possibility that autoimmunity is an aetiological factor for both diseases.

\section{Case Report}

A 27-year-old woman presented to our hospital in April 2010 with right forearm bruises of approximately $4 \mathrm{~cm} \times 3 \mathrm{~cm}$; she had no predisposing factors or history of bleeding tendency. Possible trauma was diagnosed. The next day, the bruises grew larger, and the patient sought another consultation at a private general medical centre, where the diagnosis of a possible insect bite was made. The patient was concerned about the progressively increasing size of the bruises, which reached approximately $15 \mathrm{~cm} \times 5 \mathrm{~cm}$ over 2 days. Therefore, she visited the haematology outpatient department of our hospital 3 days after the bruises were first noticed. Haematological investigations at presentation revealed normal results for liver enzymes, immunoglobul in profile and antinuclear factor. A virology screening study showed negative results for hepatitis $\mathrm{C}$, hepatitis $\mathrm{B}$ and human immunodeficiency virus. C4 level was $0.16 \mathrm{~g} / \mathrm{l}$ (normal 0.16-0.47), C3 level was $0.90 \mathrm{~g} / \mathrm{l}$ (normal 0.88-2.01), and C-reactive protein level was $5.6 \mathrm{mg} / \mathrm{l}$ (normal 0.0-8.0). The level of rheumatoid factor was $<20 \mathrm{IU} / \mathrm{ml}$ (normal 0-20). The erythrocyte sedimentation rate value was $5 \mathrm{~mm} / \mathrm{h}$ (normal 0-20). The patient's blood group was 0 positive. Coagulation profile results obtained on presentation were as follows: prothrombin time was $13.9 \mathrm{~s}$ (reference range 10.0-13.0), bleeding time was $2.10 \mathrm{~min}$ (reference range 1.00-4.00), von Willebrand factor Ag-O was $64.4 \%$ (reference range 41.1-125.9), von Willebrand factor ACTO was 48.3\% (reference range 38.0-125.2), and 
fibrinogen was $3.39 \mathrm{~g} / \mathrm{l}$ (normal 2.20-4.96). Results of the activated partial thromboplastin time (APTT) test (a before-and-after, mixed, study test) showed that prolonged APTT did not correctly adjust when adding normal plasma to patient plasma, as it would in the case of congenital haemophilia A (table 1).

The patient had a history of recurrent acquired achalasia since 2000 (aged 16 years), when she exhibited symptoms of dysphagia for solids and liquids. She presented to medical casualty 7 times with choking and difficulty swallowing. In early 2003, she underwent gastrointestinal endoscopy for the first time, which did not indicate any abnormal findings. However, barium swallow showed abnormal barium retention for more than $15 \mathrm{~min}$ at the distal oesophagus. Radiological findings

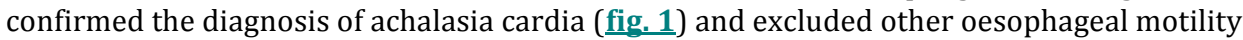
disorders. She subsequently underwent oesophageal manometry. Following the diagnosis of achalasia cardia, she was transferred to Bupa Cromwell Hospital, where further tests confirmed this diagnosis. A Heller myotomy was performed, after which the symptoms of dysphagia resolved.

In 2004, the patient relapsed and underwent balloon dilatation at Bupa Cromwell Hospital, but only partial improvement was noted. In 2005, she had recurrent episodes of dysphagia grade 3. Oesophageal manometry was repeated and revealed recurrent achalasia. In 2006, she had recurrent episodes of dysphagia during pregnancy. She was advised to undergo balloon dilatation after delivery. In 2007, she relapsed again and underwent Nissen fundoplication without success. In 2008, the achalasia relapsed, and she underwent a second balloon dilatation but experienced no improvement. In January 2010, she underwent another Nissen fundoplication, oesophageal myotomy, and a Toupet fundoplication at the Mayo Clinic in the United States. Her symptoms improved, but at presentation to our clinic in April 2010 she had arm bruises and recurrent discomfort in swallowing.

Upon diagnosis of acquired autoimmune haemophilia, the patient was administered prednisolone at a dose of $1 \mathrm{mg} / \mathrm{kg}$ ( $50 \mathrm{mg}$ ) for 1 week. She showed good response (table 2), and the dose was then tapered by reducing prednisolone by $10 \mathrm{mg}$ each week until week 4, after which time a dose of $10 \mathrm{mg}$ was maintained for 6 months. The total period of steroid therapy was 7 months. The patient's achalasia symptoms improved after initiation of corticosteroid therapy for acquired haemophilia, and her dysphagia has been in complete remission for more than 2 years without relapse.

\section{Discussion}

Human autoimmune diseases are generally more common in females than in males. Many chronic autoimmune diseases have a relapsing-remitting course. Individuals with a particular autoimmune disease have an increased risk of developing other autoimmune diseases. However, there is increasing evidence that another important genetic component increases the susceptibility to 'autoimmunity-in-general' [6]. Cross-reacting autoantibodies targeting an epitope have the potential to spread the autoimmune response to other RNA-binding proteins through molecular mimicry; subsequently, intramolecular spreading to these specific proteins can occur [7].

In the present case, the patient had chronic, relapsing, acquired achalasia from the age of 16 years. Ten years after the first manifestation of achalasia, she developed severe autoimmune acquired haemophilia $\mathrm{A}$, which was treated promptly and aggressively with high-dose steroids for 7 months. During the 7 months of steroid treatment and the nearly 2 years since cessation of treatment, the achalasia and the haemophilia A have remained in remission.

In individuals with autoimmune diseases, there is an increased risk of developing other autoimmune diseases mediated by additional or cross-reacting autoantibodies. Therefore, we assume this patient had autoimmune acquired achalasia. We believe that, 10 years after the first manifestation of achalasia, she developed another well-known autoimmune antibody against FVIII, leading to acquired haemophilia A. 
The two disorders may be targeted by a cross-reacting autoantibody; however, we have no indication of the relevant autoimmune target in achalasia. The occurrence of the two disorders in one patient suggests that achalasia is also an autoimmune disorder. This is supported by the patient's long-standing remission after steroid therapy. Moreover, achalasia has previously been reported in association with other autoimmune disorders, such as autoimmune thyrotoxicosis and autoimmune polyglandular polymyositis. Thus, autoimmune disorders can share the cross-reacting antibody [8].

The treatment of acquired haemophilia is generally similar to other autoimmune disorders and includes immunosuppressive treatment to eliminate the inhibitory antibodies. Typical treatment, based on the results of a randomised clinical trial, involves administration of prednisolone at a dose of $1 \mathrm{mg} / \mathrm{kg}$ with or without orally administered cyclophosphamide (50-100 mg/day) [9]. The use of rituximab may be considered for initial therapy in cases where the initial antibody titre is high [10]. Porcine factor VIII (Hyate: $\mathrm{C}$ ) is a safe and effective treatment for bleeding episodes associated with acquired haemophilia [11]; however, azathioprine therapy may be preferred for women of child-bearing age. Treatment should be continued for up to 6 weeks with regular patient assessment. Most patients will respond well to this treatment combination. However, relapse can occur when the drugs are discontinued or the dose is reduced. Infusions of immunoglobulin may also be beneficial. A prospective, non-randomised trial involving immunoglobulin therapy assessed the response to doses of $1.0 \mathrm{~g}$ per day for 2 days or $0.4 \mathrm{~g}$ for 5 days in patients with acquired haemophilia. A 25\% reduction in inhibitor titre was observed in 8 of 16 assessable patients [12]. However, the use of other immunosuppressive agents should also be considered.

\section{Conclusion}

Our patient experienced an almost annual relapse of achalasia cardia since the age of 16, but achieved complete remission for more than 2 years following corticosteroid therapy, which was administered for the associated severe acquired haemophilia A. This remission suggests that acquired achalasia may also be an autoimmune disorder, similar to acquired haemophilia $\mathrm{A}$, and that corticosteroids or other immunosuppressive therapy may be the appropriate treatment prior to surgical intervention. However, further observational studies are required to document this relationship, confirm this association and elucidate additional insights into the aetiology of this condition.

\section{Acknowledgement}

We would like to express our gratitude to all those who contributed to this case report. We would like to thank Ms. Zainab Bahbahani from the Coagulation Section and Saud Al-Saif from the X-Ray Department of Amiri Hospital. 
Table 1. Mixed study results

\begin{tabular}{lll}
\hline & Result & Reference range \\
\hline APTT patient, s & 62 & $25-35$ \\
APTT control, s & 36 & $25-35$ \\
P+C (pre-incubation), s & 65 & $25-35$ \\
P+C (2 h post-incubation), s & 88 & $25-35$ \\
\hline
\end{tabular}

Table 2. Normal values for APTT (25-35 s), FVIII Act (50-150\%) and VW Act (38.2-125.2\%)

\begin{tabular}{lllllll}
\hline Duration & INR & APTT & Mixed & FVIII Act & VW Act & VW Act \\
\hline At presentation & 1.24 & 71 & 40 & - & - & \\
After 1 day & 1.07 & 62 & 42 & 1.98 & 48.3 & 64.4 \\
After 2 weeks & 1.07 & 40 & 36 & 39.80 & - & \\
After 3 months & 1.19 & 29 & - & 144 & - & \\
\hline
\end{tabular}

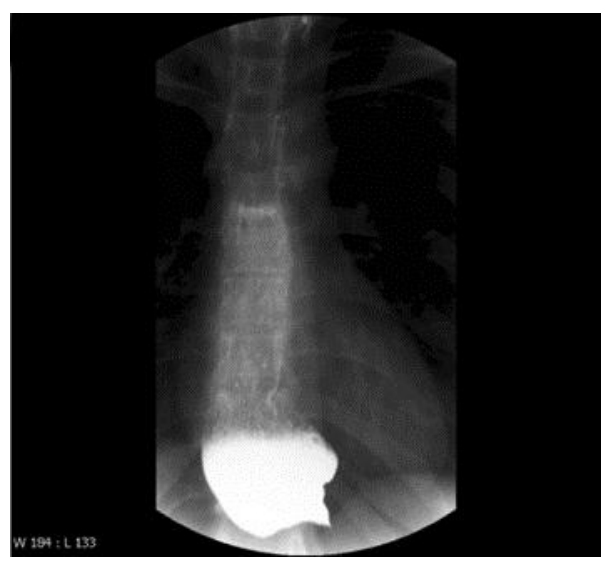

Fig. 1. Barium meal study showing significant blockage at the lower oesophagus just above the gastro-oesophageal junction. There is evidence of a small, tapering, rat-tail appearance associated with dilatation of the oesophagus lumen with marked fluid level. No extraluminal leak was noted.

\section{References}

1 Green D: The management of acquired haemophilia. Haemophilia 2006;12(suppl 5):32-36.

2 Jamil A, Bayoumy M, Iram D, Adler B: Pediatric severe hemophilia: initial presentation, characteristics and complications. Internet J Hematol DOI: $10.5580 / \mathrm{d} 14$.

- Ellis FG: The aetiology and treatment of achalasia of the cardia. Ann R Coll Surg Engl 1962;30:155-182.

4 Farrokhi F, Vaezi MF: Idiopathic (primary) achalasia. Orphanet J Rare Dis 2007;2:38.

5 Clark SB, Rice TW, Tubbs RR, Richter JE, Goldblum JR: The nature of the myenteric infiltrate in achalasia: an immunohistochemical analysis. Am J Surg Pathol 2000;24:1153-1158. 
6 Pender MP: Epstein-Barr virus and autoimmunity: in Shoenfeld Y, Noel R, Rose NR (eds): Infection and Autoimmunity. Amsterdam, Elsevier, 2004, pp 163-170.

7 Routsias JG, Tzioufas AG: Epitopes of autoantigens and their role in initiation and propagation of systemic autoimmunity. Eur Musculoskelet Rev 2009;4:51-55.

-8 Moses PL, Ellis LM, Anees MR: Antineuronal antibodies in idiopathic achalasia and gastro-oesophageal reflux disease. Gut 2003;52:629-636.

9 Giangrande P: Acquired Hemophilia. Treatment of Hemophilia. Oxford, World Federation of Hemophilia, 2005, p 38

10 Aggarwal A, Grewal R, Green RJ, Boggio L, Green D, Weksler BB, Wiestner A, Schechter GP: Rituximab for autoimmune haemophilia: a proposed treatment algorithm. Haemophilia 2005;11:13-19.

11 Morrison AE, Ludlam CA, Kessler C: Use of porcine factor VIII in the treatment of acquired hemophilia. Blood 1993;81:1513-1520.

12 Schwartz RS, Gabriel DA, Aledort LM, Green D, Kessler CM: A prospective study of treatment of acquired (autoimmune) factor VIII inhibitors with high-dose intravenous gammaglobulin. Blood 1995;86:797-804. 